\title{
September 2014 Arizona Thoracic Society Notes
}

The September 2014 Arizona Thoracic Society meeting was held on Wednesday, 9/24/14 at the Kiewit Auditorium on the University of Arizona Medical Center campus in Tucson beginning at 5:30 PM. This was a dinner meeting with case presentations. There were about 21 in attendance representing the pulmonary, critical care, sleep, pathology and radiology communities.

Four cases were presented:

1. Mohammad Dalabih presented a 22 year old hypoxic man with a history of asthma and abdominal pain. A bubble echocardiogram showed bubbles in the left ventricle within 3 heartbeats. Thoracic CT scan showed a pulmonary arteriovenous malformation (AVMs). The patient underwent coil embolization and improved. Dr. Dalabih reviewed the diagnosis and management of pulmonary AVMs (1).

2. Aarthi Ganesh presented a 70 year old woman complaining of dyspnea on exertion. A chest $\mathrm{x}$-ray showed complete opacification of the right hemithorax and a thoracic CT scan showed a large right pleural effusion with right lung atelectasis. After thoracentesis was nondiagnostic, she underwent video-assisted thorascopic surgery (VATS). Although she clinically appeared to have mesothelioma, histology was consistent with a pseudomesotheliomatous adenocarcinoma. She is currently undergoing treatment with platinum based agents.

3. Gordon Carr presented a 75 year old woman with dyspnea. Chest x-ray showed interstitial disease with a possible usual interstitial pneumonia (UIP) pattern on CT scan. Dr. Carr reviewed the initial evaluation and diagnosis of the interstitial lung disease (2). VATS showed a bronchocentric process with some fibrosis in the periphery most consistent with chronic hypersensitivity pneumonitis. The likely source was thought to be mold in her indoor pool area.

4. James Knepler presented a 55 year old woman with breast cancer and bone metases receiving tamoxifen. She also had a history of multiple sclerosis and was receiving on interferon-beta 1a. A positron emission tomography (PET) scan showed increased uptake in several mediastinal lymph nodes. Endobronchial ultrasound (EBUS) guided aspiration biopsy was non-diagnostic. Endobronchial biopsy showed granulomas. It was felt the most likely diagnosis was interferoninduced sarcoidosis. Several case reports have recently been published.

There being no further business the meeting was adjourned about 7:00 PM. The next meeting will be Phoenix on Wednesday, October 22, 6:30 PM at Scottsdale Shea Hospital.

Richard A. Robbins, MD

References 
1. Gossage JR, Kanj G. Pulmonary arteriovenous malformations. A state of the art review. Am J Respir Crit Care Med. 1998;158(2):643-61. [CrossRef] [PubMed]

2. Selman M, Pardo A. Update in diffuse parenchymal lung disease 2012. Am J Respir Crit Care Med. 2013;187(9):920-5. [CrossRef] [PubMed] 